\title{
The Application of the Wavelet Noise Reduction in Gear Box Fault Diagnosis
}

\author{
Jinyu Wang ${ }^{1, \mathrm{a}}$, Dejian Kong ${ }^{2, \mathrm{~b}}$, Longying Zhao ${ }^{3, \mathrm{c}}$, Dongxue Lin $^{4, \mathrm{~d}}$ \\ ${ }^{1,2}$ Department of Electrical \& Information Engineering, Northeast Petroleum University, Heilongjiang \\ Daqing, 163318, China \\ ${ }^{3}$ The First Electrical Team of Electrical Maintenance of the Fourth Oil Production in Daqing 163318, \\ China \\ aemail: wangjydxI@126.com, 'email: kongdejian0154@163.com
}

Keywords: Soccer Robot; Mechanical Analysis; Optimal Design

\begin{abstract}
With the QPMZ - II type gear box as experimental object, according to the failure mechanism and vibration characteristics under actual condition of the gear box, the vibration signals are decomposed into components at different frequency bands and time period by wavelet transform, so that the interference signals and the desired signals are located in different frequency bands. The wavelet coefficient corresponded by interference signals is set to zero, reconstruct the signals, then the signals are obtained after the noise reduction. Analysis the envelope power spectrum can analysis the diagnose failure effectively.
\end{abstract}

\section{Introduction}

In the fault diagnosis of mechanical equipment, when the fault message is stationary signals, generally use the characteristics of the signals determined and the noise characteristics, then take the method of Fourier analysis to achieve the purpose of de-noising. But the signals in practical engineering applications, which is often non-stationary signals. With the traditional Fourier transform method cannot satisfy the requirement of non-stationary signals filtering pre-processing. This would require the use of wavelet analysis. Wavelet analysis is a new time-frequency analysis method, which inherits the Fourier analysis with the thought of harmonic function to approximate arbitrary signals. Just the basic function of wavelet analysis is a series of scale variable functions. This makes the wavelet analysis has a good time-frequency localization characteristics and the adaptive capacity of signals. It can decompose the time-varying signals effectively . Providing a new and powerful analytical tool of fault diagnosis for the control system. Wavelet transform is a linear transformation, no distractions, has high frequency resolution at low frequency and high time resolution at high frequencies. Time and frequency resolution is variable. It has zoom feature. Especially, it is particularly effective for analysising the signal singularity, the location of singularity and the size of the singular degree. It can analysis the high-frequency signal and the short-term component in the time domain and the frequency domain. The fault characteristic signal can be separated effectively. Therefore, using wavelet transform to eliminate noise signal is feasible.

\section{The application of wavelet analysis in signal decomposition}

Using wavelet decomposition to establish the model for noise reduction of the time series. First, analysis the gaussian white noise signal. White noise signal is shown in figure 1 . According to the Mallat pyramidal decomposition algorithm, using Daubechies wavelet function, the signal divided into different frequency channel, and the components of each frequency channel are decomposed by phase. The higher of the frequency, the finer of the phase division, conversely more sparsely. Decompose the gaussian white noise signal in five layer through the wavelet transform. The sampling frequency of the original signal is 1024. Figure 2 shows the results of the fifth layer decomposition. 


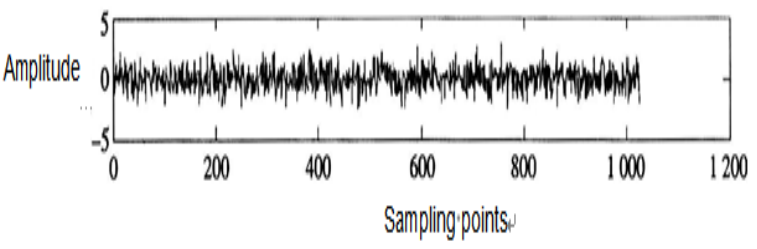

Fig.1.Gaussian white noise

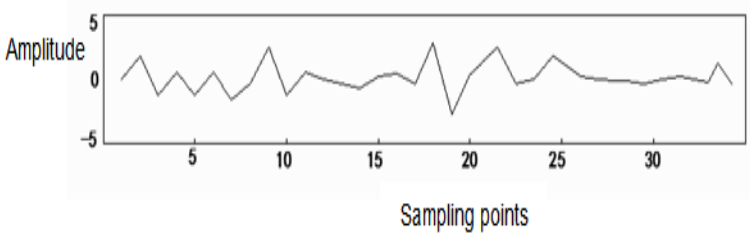

Fig.2.Result of the fifth layer decomposition

Based on the Mallat pyramidal decomposition algorithm, decompose the general time series in five layer through the wavelet transform. The sampling frequency of the original signal is remain 1024. As shown in Figure 3, Figure 4.

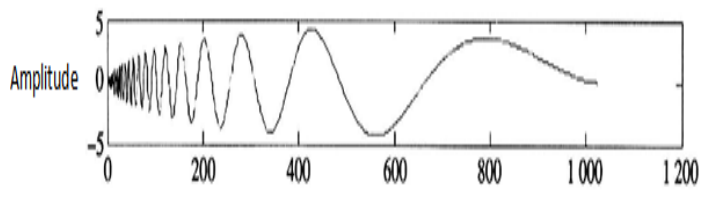

Sampling points

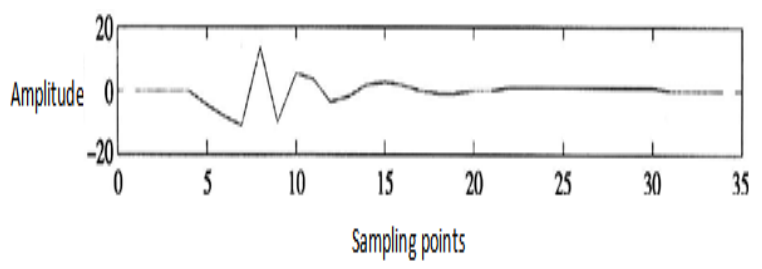

Sampling points

Fig.3.General time series signal Fig.4.The fifth layer of general time-series decomposition

Comparing figure 2 and figure 4, you can see that the results of white gaussian noise and general time-series are different. Gaussian white noise' amplitude are homogeneous in each wavelet decomposition, but the decomposition coefficient of the general time-series are larger in some point. Based on this, we can make use of wavelet decomposition to get rid of the white gaussian noise signal.

\section{Establish Wavelet Noise Reduction Model}

Assuming that a signal with noise

$y_{i}=f_{i}+e_{i}$

$e_{i}$ in the formula is the gaussian white noise which variance is $\sigma^{2}$, that is $\left(0, \sigma^{2}\right)$. Make $f_{i}^{\prime}$ as $f_{i}$ 's estimate, then the average variance of construct estimation model $f_{i}^{\prime}$ is,

$$
E \boxminus\left(f_{i}^{\prime}-f\right) \square^{2}=\sum_{i=1}^{n}\left(f_{i}^{\prime}-f\right)^{2}
$$

White noise $e_{i}$ affects all wavelet coefficients, but $f_{i}$ only affects a few wavelet coefficients. Setting a threshold value $\lambda$, each coefficient which below the threshold value set to zero. The wavelet coefficients which higher than $\lambda$ is the estimated value of signal $f_{i}$. So the wavelet noise reduction model can be described as follows.

1) Calculating the wavelet coefficients $w_{j k}$ of signal $y_{i}$.

2) Calculation the threshold value.

$$
\lambda=\sqrt{2 \lg (n)}
$$

$\mathrm{N}$ is the length of the input sample type.

If $|y| \geq \lambda$, then

$$
n_{\lambda}=\operatorname{sign}(y)(|y|-\lambda)
$$

Put $w_{j k}$ into the equation (4), obtain the estimate value of wavelet coefficients $\theta=n_{\lambda}\left(w_{j k}\right)$.

3)Do wavelet reconstruction for $\theta$,obtain the estimated signal $f\left(t_{i}\right)=W^{T} \theta$ of $f\left(t_{i}\right)$, that is the signal after the noise reduction. $W$ is the wavelet decomposition operator.

The wavelet noise reduction model described above, the wavelet coefficients is $\theta=W^{T} f$, then the estimate value of average variance $f\left(t_{i}\right)$ is

$$
E \boxminus\left(f_{i}^{\prime}-f\right) \square^{2}=E \boxminus\left(\theta^{\prime}-\theta\right) \square^{2}=\sum_{i=1}^{n}\left(\theta_{i}^{\prime}-\theta\right)^{2}
$$


Thus, the estimation error of $f\left(t_{i}\right)$ depending on the value of $\theta$, and the ideal estimate values of $\theta$ is $\theta$ which obtained by the formula (4). Therefore by the formula (5) can obtain the desired estimate of the mean square sense.

\section{Gearbox fault analysis and diagnosis}

According to the wavelet noise reduction model, By monitoring the position and amplitude of modulus maxima to complete the characterization and analysis of the signal. Identify the mutant signal from the vibration signal that mixed with noise efficiently. That is able to take advantage of the difference between signal and noise in the time domain and frequency domain simultaneously, implement signal-to-noise separation efficiently, obtain ideal effect of noise reduction, so as to achieve condition monitoring and fault diagnosis of gearbox. In this experiment, QPMZ-II type closed gear box as the research object, its internal structure model shown in Figure 5, the bearing parameters in Table 1. Gear is one of the most complex mechanical parts in mechanical processing equipment, since the sensor is difficult to install to the failure parts. Gear radial vibration is transmitted through the bearing to the bearing housing, causing vibration bearing. The radial vibration contains a wealth of gear fault information, using accelerometers sensors mounted on the bearing housing to pick up the vibration signals of the bearing.

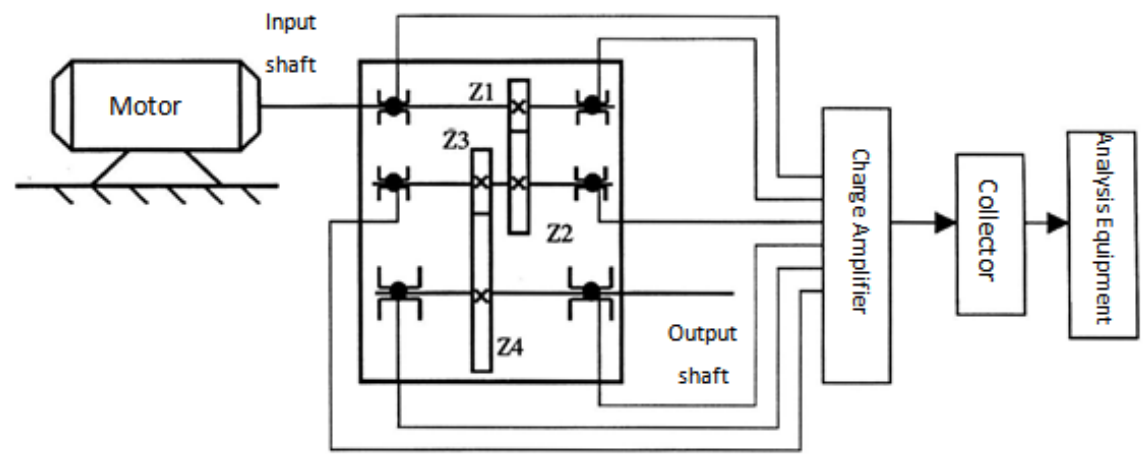

Fig.5.Gearbox model

Table 1 Gearbox bearing parameters

\begin{tabular}{ccccccc}
\hline \multirow{2}{*}{ Model } & Inner diameter & Outer diameter & Ball diameter & Festival Trail & The number & The contact angle \\
& $r / \mathrm{mm}$ & $R / \mathrm{mm}$ & $d / \mathrm{mm}$ & $D / \mathrm{mm}$ & of balls $/$ & $\begin{array}{c}\text { Months } \\
6406\end{array}$ \\
6 & 15 & 45 & $19 \circ 05$ & 60 & 6 & 0 \\
\hline
\end{tabular}

The input shaft speed of gearbox is adjusted to 780r / min, collecting the vibration signal of gearbox, Figure 6 shows the raw vibration signal with noise. Using the established model of wavelet noise reduction to analysis the vibration signal of gearbox. The signal after the noise reduction is shown in Figure 7. Compare Figures 6 and 7,we can see that, wavelet transform is used to analyse the de-noising processing of vibration signal, without distortion, making data preprocessing of fault signal, retain the failure impact signal of gear box. In order to extract the failure frequency of gear box, done Hilbert envelope and spectral analysis for the vibration signal which noise was reducted, the results shown in Figure 8. From the power spectrum analysis, the frequency of $338.1 \mathrm{~Hz}$ can be found. While the fault characteristic frequency of the bearing is the frequency of outer ring and its harmonics. Theoretical calculation, by contrast shows that the outer ring of the bearing has pitting failure.

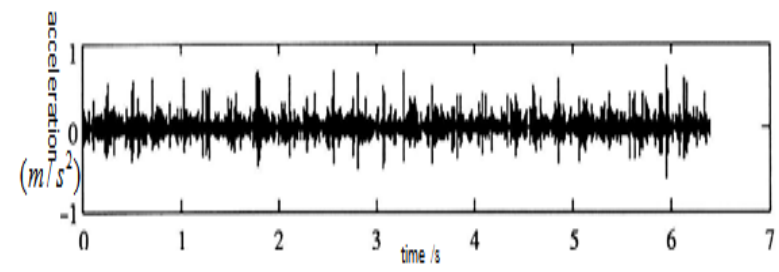

Fig.6.Vibration signal containing noise

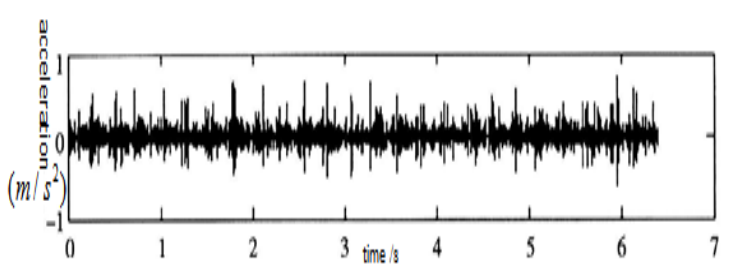

Fig.7.Vibration signal after the noise reduction 


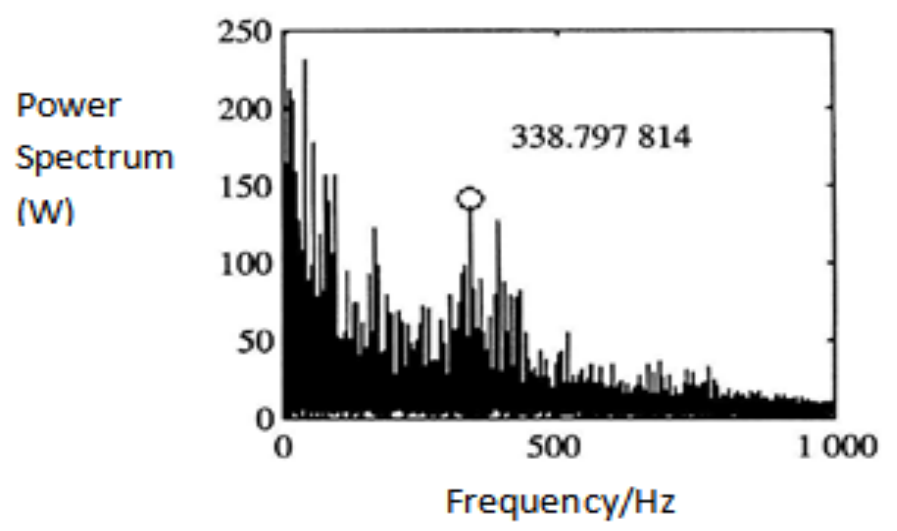

Fig.8.Signal envelope spectrum after noise reduction

\section{Conclusion}

In the fault analysis and diagnosis of gear box, using vibration sensor mounted on the bearing housing to measure the vibration signals of the bearing. If using the traditional method of power spectral analysis, then the vibration signal is very complex, some weak fault signal is often submerged. Using the wavelet transform to reduce the noise of original signal, and then do reconfigurable operation. It can improve the ratio of signal to noise and obtain high resolution. Getting the original signal for analysis quickly. Then do the envelope spectrum, detect the components of fault signal in the bearing. Verified by the test, it can diagnose some typical failure modes of gearbox effectively. And has a higher diagnostic accuracy than traditional method.

\section{References}

[1] Rezaei A, Dadouche A. Development of a turbojet engine gearbox test rig for prognostics and health management [J], Mechanical Systems and Signal Processing, 2012, 33(0): 299-311.

[2] Eftekharnejad B, Addali A, Mba D. Shaft crack diagnostics in a gearbox [J], Applied Acoustics, 2012, 73(8): 723-733.

[3] Bartelmus W, Chaari F, Zimroz R. Modelling of gearbox dynamics under time-varying nonstationary load for distributed fault detection and diagnosis [J], European Journal of Mechanics A/Solids, 2010, 29(4): 637-646.

[4] Omar F K, Gaouda A M. Dynamic wavelet-based tool for gearbox diagnosis [J]. Mechanical Systems and Signal Processing, 2012, 26(0): 190-204.

[5] Mohamed M H. Rules extraction from constructively trained neural networks based on genetic algorithms [J]. Neurocomputing, 2011, 74(17): 3180-3192.

[6] Lin Jinshan. Gearbox Fault Diagnosis based on EEMD and Hilbert transform [J]. Mechanical Transmission, 2010, (05): 62-64. 